\title{
Griseofulvin solvate solid dispersions with synergistic effect against fungal biofilms
}

\section{Article}

\section{Accepted Version}

Creative Commons: Attribution-Noncommercial-No Derivative Works 4.0

Al-Obaidi, H., Kowalczyk, R. M., Kalgudi, R. and Zariwala, M. G. (2019) Griseofulvin solvate solid dispersions with synergistic effect against fungal biofilms. Colloids and Surfaces B: Biointerfaces, 184. 11540. ISSN 09277765 doi: https://doi.org/10.1016/j.colsurfb.2019.110540 Available at https://centaur.reading.ac.uk/86565/

It is advisable to refer to the publisher's version if you intend to cite from the work. See Guidance on citing.

To link to this article DOI: http://dx.doi.org/10.1016/j.colsurfb.2019.110540

Publisher: Elsevier

All outputs in CentAUR are protected by Intellectual Property Rights law, including copyright law. Copyright and IPR is retained by the creators or other copyright holders. Terms and conditions for use of this material are defined in the End User Agreement.

\section{www.reading.ac.uk/centaur}

\section{CentAUR}

Central Archive at the University of Reading 
Reading's research outputs online 


\section{Journal Pre-proof}

Griseofulvin solvate solid dispersions with synergistic effect against fungal biofilms

Hisham Al-Obaidi, Radoslaw M. Kowalczyk, Rachith Kalgudi, Mohammed Gulrez Zariwala

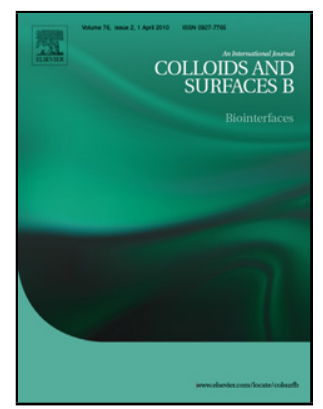
PII:
S0927-7765(19)30684-8
DOI:
https://doi.org/10.1016/j.colsurfb.2019.110540
Reference:
COLSUB 110540

To appear in:

Colloids and Surfaces B: Biointerfaces

Received Date:

1 August 2019

Revised Date:

27 September 2019

Accepted Date:

29 September 2019

Please cite this article as: Al-Obaidi H, Kowalczyk RM, Kalgudi R, Zariwala MG, Griseofulvin solvate solid dispersions with synergistic effect against fungal biofilms, Colloids and Surfaces B: Biointerfaces (2019), doi: https://doi.org/10.1016/j.colsurfb.2019.110540

This is a PDF file of an article that has undergone enhancements after acceptance, such as the addition of a cover page and metadata, and formatting for readability, but it is not yet the definitive version of record. This version will undergo additional copyediting, typesetting and review before it is published in its final form, but we are providing this version to give early visibility of the article. Please note that, during the production process, errors may be discovered which could affect the content, and all legal disclaimers that apply to the journal pertain.

(c) 2019 Published by Elsevier. 
Griseofulvin solvate solid dispersions with synergistic effect against fungal biofilms

Running title: Griseofulvin solvates against fungal biofilms

Hisham Al-Obaidi *1, Radoslaw M. Kowalczyk', Rachith Kalgudi, Mohammed Gulrez Zariwala ${ }^{3}$

${ }^{1}$ The School of Pharmacy, University of Reading, Reading RG6 6AD, UK

${ }^{2}$ Chemical Analysis Facility, University of Reading, Reading RG6 6AD, United Kingdom

3 Faculty of Science \& Technology, University of Westminster, $115 \mathrm{New}$ Cavendish Street, London, W1W 6UW, United Kingdom

${ }^{*}$ Corresponding author:

h.al-obaidi@reading.ac.uk

\section{Graphical abstract}

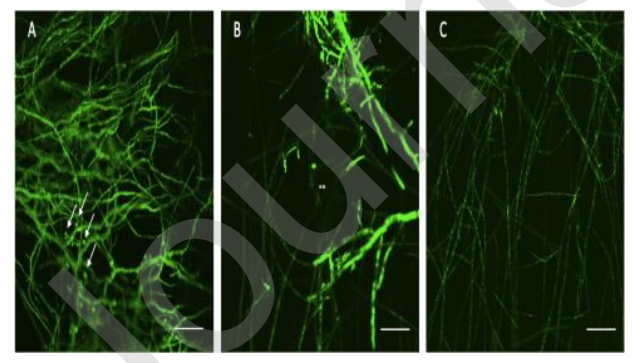

Highlights

- Novel polymeric dispersions were prepared using the solvate form of griseofulvin

- Enhanced antifungal properties were found for the dispersions prepared using HPMCAS

- A correlation between the interactions in the solid and liquid was found

- Formed solvates attach to the biofilms interface via hydrogen bonding 


\section{Abstract}

Fungal biofilms are invariably recalcitrant to antifungal drugs and thus can cause recurrent serious infections. The aim of this work was to prepare highly effective form of the antifungal drug griseofulvin using the chloroform solvate embedded into different polymeric matrices. Based on their solid solubility, solvated (chloroform) and non-solvated (methanol and acetone) solid dispersions were prepared using different materials: silica, microcrystalline cellulose, polyvinylpyrrolidone and hydroxypropyl methylcellulose acetate succinate (HPMCAS) by which HPMCAS dispersions showed the highest solubility of about $200 \mu \mathrm{g} / \mathrm{mL}$ compared with $\sim 30 \mu \mathrm{g} / \mathrm{mL}$ for pure griseofulvin. The anti fungal potential of griseofulvin was assessed against the dermatophytes T. rubrum. Metabolic and protease activity of T. rubrum NCPF 935 with and without the presence of GF:HPMCAS chloroform solvates showed significant reduction compared to the untreated control after $24 \mathrm{~h}$ period. Confocal laser scanning microscopy showed thin hyphae compared to Control and GF:HPMCAS (non solvated). Dynamic vapour sorption data showed that HPMCAS formed most stable solvate structure preventing recrystallization and solvate expulsion, which could explain the disruptive effect of the biofilms. This could be explained by the formed hydrogen bonds as revealed by the solid and liquid state NMR data, which was further confirmed via thermal and FTIR analyses.

Keywords: Fungal biofilms, solvates, saturation solubility, thermal analysis 


\section{Introduction}

Biofilms are surface-associated microbial communities that exist within a selfsecreted matrix consisting primarily of polysaccharides, proteins and nucleic acids forming the biofilm extracellular matrix (ECM) [1, 2]. Biofilm structures formed by fungi may present favourable conditions for survival within an infected host [3]. Dermatophytes such as T. rubrum, keratinolytic, saprophytic fungi, are known to form biofilm and commonly implicated as a common cause for athlete's foot and fungal infection of the nails [4]. Griseofulvin is one of the most commonly used antimicrobials to treat dermatophytosis caused by T. rubrum [5] nevertheless, there have been reports of increased incidence of resistance to griseofulvin, which makes treatment with griseofulvin less effective $[6,7]$. This could be attributed to the biofilm formation and longer duration needed to complete the treatment.

The low aqueous solubility of griseofulvin makes its formulation particularly challenging for oral drug delivery, which results in larger doses needed to reach a therapeutic level. Hence, the challenge in improving the antifungal activity of griseofulvin is two folds; first to improve its aqueous solubility and secondly to overcome resistance due to biofilm formation. Crystal modifications of drug forming metastable drug polymorphs, hydrates, and cocrystals have been among various approaches to improve aqueous solubility of drugs [8-10]. 
Solvates (also known as pseudopolymorphs) comprise of solvent-drug crystals, which have completely different structural configuration to the parent drug crystals [11]. Recently, it has been shown that ethanol solvates can significantly improve the dissolution of pioglitazone with potential enhancement of in vivo levels in beagle dogs [12]. It is generally agreed that solvates can form through either adsorption to the crystal cell surface or though occupying voids within imperfect crystals [13]. It was shown recently that dextran shell nanoparticles eradicated biofilms of various multidrugresistant Gram-positive bacteria [14]. This effect was attributed to improved solubility of the bacteria-nanoparticles complex due to the presence of the dextran shell [14]. Based on chloroform solvate, the aim of this work was to prepare a highly synergistic solvate form of griseofulvin able to diffuse across the biofilms and enhance their solubility. The solvate form is stabilised within a polymeric matrix, which will also help to enhance the aqueous solubility of the embedded drug.

Griseofulvin (GF) was embedded within different matrices, namely silica, microcrystalline cellulose (MCC), polyvinylpyrrolidone (PVP) and hydroxypropyl methylcellulose acetate succinate (HPMCAS). GF and the polymer dispersions were prepared using acetone, methanol and chloroform as solvents. They were selected because of their distinct properties in terms of hydrogen bonds formation, boiling point and polarity. Chloroform and methanol are Lewis acids with unique properties of chloroform to form $\mathrm{Cl}$...Cl interactions while acetone is Lewis base with distinct proton accepting capability. By using different matrices, it was possible to evaluate the impact 
of drug excipient interactions on crystallization behaviour. Silica is amorphous that lacks hydrogen bonding ability, PVP is a Lewis base amorphous polymer able to accept hydrogen bonds, MCC is partially crystalline able to accept and donate hydrogen bonds while HPMCAS is amorphous polymer and hydrogen bond acceptor and donor.

\section{Experimental Section}

\subsection{Materials}

Griseofulvin 97\%, silicon dioxide nanopowder 99.5\%, microcrystalline cellulose (MCC) and polyvinylpyrrolidone (PVP) K30 were purchased from Sigma-Aldrich (Dorset, UK). Hydroxypropyl methylcellulose acetate succinate (HPMCAS) was obtained from Shin-Etsu Chemical Co. Ltd (Tokyo, Japan). Acetone, chloroform and methanol were purchased from Fisher Scientific (Loughborough, UK). Ethanol, potassium dihydrogen phosphate, KH2PO4 and sodium hydroxide, $\mathrm{NaOH}$ pellets were obtained from VWR International (Leicestershire, UK).

\subsection{Dynamic vapor sorption}

Solid dispersions of GF (10 mg) were weighed at $25{ }^{\circ} \mathrm{C}$ using a Dynamic Vapor Sorption analyzer (SMS, UK). Two sources of nitrogen gas were used where one of them is a dry gas while the second is $100 \%$ moist. By mixing both of them, the desirable relative humidity was obtained. The variation in 
weight was recorded using a two-arm balance where the reference pan was empty and the sample pan was filled with the sample. Nitrogen gas was passed over the sample to remove the residual solvent content (using $0 \%$ relative humidity). Once this was achieved, nitrogen gas is passed with the desired relative humidity $(\mathrm{RH})$.

\subsection{Differential Scanning Calorimetry (DSC)}

Thermal analysis was performed on a 2920 TA Instruments (Hertfordshire, UK) differential scanning calorimeter calibrated with indium using a purge of nitrogen gas at $20 \mathrm{~mL} / \mathrm{min}$. A pinhole was created on an aluminium lid (TA instruments) to remove residual solvent. $8-10 \mathrm{mg}$ of sample was loaded into an aluminium pan and hermetically sealed. The samples were scanned from $10^{\circ} \mathrm{C}$ to $250^{\circ} \mathrm{C}$ using a heating rate of $10^{\circ} \mathrm{C} / \mathrm{min}$. The melting enthalpy, onset and peak melting temperature were recorded and their values represent an average value of triplicates.

\subsection{Fourier transform infrared spectroscopy (FTIR)}

FTIR was performed on the prepared dispersions to examine potential hydrogen bonding between GF and the polymers. The infrared spectra of samples were obtained using Perkin Elmer Spectrum One FTIR spectrometer, equipped with a diamond attenuated total reflectance (ATR) accessory (Shelton, Connecticut, USA). A total of 64 scans were collected for each sample with a resolution of $4 \mathrm{~cm}-1$ from a frequency range of $4000 \mathrm{~cm}-1$ to $550 \mathrm{~cm}-1$. 


\subsection{Saturation solubility measurements}

From each solid mixture quantities equivalent to $5 \mathrm{mg}-10 \mathrm{mg}$ equivalent of griseofulvin was weighed. $1 \mathrm{~mL}$ of phosphate buffer was added into the microtube which was then loaded on a Stuart SB2 rotary mixer (Staffordshire, UK) and mixed for $1,3,5$ and $72 \mathrm{~h}$. This was followed by centrifugation for 10 min at 13000 RPM on a Heraeus Biofuge Pico 24-place microliter centrifuge (Thermo Scientific, Hertfordshire, UK). The supernatant was separated and the UV absorbances of the supernatants were measured using a Thermo Scientific Spectronic spectrophotometer (Thermo Scientific, Waltham, USA) using a wavelength of $295 \mathrm{~nm}$. Absorbance values were converted into solubility values using standard calibration curve of griseofulvin. Solubility studies were conducted in triplicates and the average and standard deviations were calculated.

\subsection{Temperature controlled X-ray powder diffraction}

The polymorphic nature of the formed samples was studies using $\mathrm{x}$-ray powder diffraction (XRPD). All samples were scanned using a Bruker D8 advance X-ray diffractometer (Bruker AXS GmbH, Germany) which is a Cusource, theta- theta diffractometer equipped with a Lynx eye position sensitive detector. It was operated at $40 \mathrm{kV}$ generator voltage and $40 \mathrm{~mA}$ generator current. The samples were analysed using DFFRAC plus XRD commander software (Bruker AXS GmbH, Germany) with a $2 \theta$ range of 5-45, a step size of $0.02^{\circ}$ and time per step of $1.33 \mathrm{~s}$. Glass Capillary x-ray 
diffraction data were collected on Bruker D8 Advance Material lightly ground and packed in $0.7 \mathrm{~mm}$ capillary, which was then heated with a co-axially, mounted cryostream. Compact controller set to ramp to temperature, then held for 5 minutes to allow the capillary to warm up, before collecting data. This was followed by a ramp to next temperature, hold, collect cycle within a temperature range of $30-200{ }^{\circ} \mathrm{C}$.

\subsection{Solid and liquid state NMR}

Solid and liquid state NMR studies were used to investigate structural changes of GF within the solvated and non-solvated polymeric matrices. ${ }^{1} \mathrm{H}$ and ${ }^{13} \mathrm{C}$ NMR spectra of GF chloroform- $d$ solution were recorded on Bruker Avance III spectrometer (Bruker, Germany) operating at Larmor frequency of $500 \mathrm{MHz}(11.75 \mathrm{~T})$ and $125.78 \mathrm{MHz}$, respectively. The 32 transient were recorded and average into each ${ }^{1} \mathrm{H}$ spectrum. The proton decoupled ${ }^{13} \mathrm{C}$ experiment required 1024 transients, and was recorded with $8 \mathrm{sec}$ relaxation delay. The selected 2D experiments (e.g. COSY, TOCSY, HSQC and HMBC) were also recorded to confirm the assignments of the GF peaks to the molecular structure (data not shown). All spectra were referenced using TSP signal at 0 ppm.

The ${ }^{13} \mathrm{C}$ solid-state cross-polarization magic angle spinning (CPMAS) NMR spectra were recorded on Bruker Avance III spectrometer (Bruker, Germany) operating at Larmor frequency of $125.78 \mathrm{MHz}(11.75 \mathrm{~T})$. The standard bore MAS probe was used in conjunction with $4 \mathrm{~mm}$ diameter zirconia rotors, which were spun at $10 \mathrm{kHz}$ rate. The $\mathrm{CP}$ contact time was $2.0 \mathrm{~ms}$, and the $90^{\circ}$ 
pulse width was 3.7 us at a power level of 32 W. Between 256 (for GF and HPMCAS) and 1024 (all other samples) signal transients were accumulated with $6 \mathrm{sec}$ relaxation delays at ambient temperature. All spectra were referenced to external adamantane signal as a secondary reference (frequency peak at $38.0 \mathrm{ppm}$ ).

\subsection{Preparation of drug-polymer solvates and non-solvated counterparts}

Fixed weight ratios (w/w ratios) of GF and polymer, i.e. silica, microcrystalline cellulose (MCC), polyvinylpyrrolidone (PVP) and hydroxypropyl methylcellulose acetate succinate (HPMCAS) were weighed and fully dissolved in solvent of choice (acetone, methanol or chloroform) which was then removed using rotary evaporator (Buchi Rotavapor RE-111, Oldham, UK) with a set water bath temperature of $40{ }^{\circ} \mathrm{C}$. The solid remained in the flask was transferred to another container and placed under vacuum to be dried for $24 \mathrm{~h}$ to eliminate any residual solvent. The resultant solid was coground for $10 \mathrm{~min}$ and sieved via $80 \mu \mathrm{m}$ sieve.

\subsection{Preparation of fungal inoculum}

In order to study differences among prepared formulations, in vitro studies were carried out using fungal biofilms. T. rubrum NCPF 935 (PHE, UK) inoculum was prepared from initial growth on potato dextrose agar (PDA) (Sigma-Aldrich, UK) as mentioned by Brilhante et al., 2017 with a few modifications [15]. Briefly, $39 \mathrm{~g} / \mathrm{L}$ of the solids were fully dissolved in $1 \mathrm{~L}$ of distilled water and then sterilised at $121^{\circ} \mathrm{C}$ for $15 \mathrm{~min}$. The molten agar was 
poured into petri plates once it had cooled down to $\sim 55^{\circ} \mathrm{C}$ and allowed to set. A small piece of $T$. rubrum NCFP 935 was excised from the stock and placed in the centre of the PDA plates and allowed to grow for a period of 7 10 days at $28{ }^{\circ} \mathrm{C}$ for optimal sporulation. The conidia and hyphal fragments were harvested by covering the cultures with $5 \mathrm{~mL}$ of sterile $0.9 \%$ saline solution and by passing a sterile swab over the surface of the cultures. The $0.9 \%$ saline solution containing the conidia and hyphal fragments was then collected and allowed to settle for $5 \mathrm{~min}$ at $28^{\circ} \mathrm{C}$ to sediment the hyphae and separate the conidia. The conidium suspension was then gently aspirated and transferred to fresh sterile tubes and serially diluted to obtain a suspension containing $\sim 5 \times 10^{6} \mathrm{cfu} / \mathrm{mL}$. This was based on cell count performed using a haemocytometer. The conidia suspension was then diluted with $0.9 \%$ saline solution to obtain a concentration of $\sim 1 \times 10^{6} \mathrm{cfu} / \mathrm{mL}$ for biofilm formation and subsequent experiments.

\subsection{Working stock of griseofulvin formulations for biofilm assay}

GF:HPMCAS formulations were initially dissolved in DMSO (dimethyl sulfoxide) (Sigma-Aldrich, UK) according to the CLSI broth microdilution protocol (Wayne, 2008) to a concentration of $800 \mu \mathrm{g} / \mathrm{mL}$. The formulations were then further diluted in RPMI 1640 medium, buffered to $\mathrm{pH} 7$ with MOPS to obtain the desired concentrations for biofilm formation assay.

\subsection{T. rubrum NCPF 935 biofilm formation assay}

Biofilm formation by $T$. rubrum NCPF 935 was performed based on the method as mentioned by dos Santos and Dias-Souza, 2017; Costa-Orlandi et 
al., 2014 with a few modifications [16]. Briefly, $250 \mu \mathrm{L}$ of the pre-adjusted $(\sim 1$ $\times 10^{6} \mathrm{cfu} / \mathrm{mL}$ ) conidia suspension was added to 96 -well plates as required. The plates were then incubated at $37^{\circ} \mathrm{C}$ for $3 \mathrm{~h}$ for adhesion of the conidia to the surface of the wells under static conditions. Subsequently, the growth suspension was replaced with pre-sterilised $250 \mu \mathrm{L}$ of RPMI $1640(\mathrm{pH} 7)$ medium containing GF:HPMCAS (chloroform solvate) and non solvated (prepared from acetone). The concentration of the formulations used in this study were equivalent to the MIC of griseofulvin against $T$. rubrum $(8 \mu \mathrm{g} / \mathrm{mL})$, which were prepared following CLSI (the clinical and laboratory standards institute guidelines) M38-A2 for fungal work. Biofilm of T. rubrum NCPF 935 was grown with and without the addition of the formulation and grown for a period of $96 \mathrm{~h}$ at $37^{\circ} \mathrm{C}$ prior to carrying out subsequent assays.

\subsection{Quantification of T. rubrum NCPF biofilm by safranin staining}

Biofilm formation by $T$. rubrum NCPF 935 was performed based on previous method with a few modifications [16, 17]. Briefly, $250 \mu \mathrm{L}$ of the pre-adjusted $\left(\sim 1 \times 10^{6} \mathrm{cfu} / \mathrm{mL}\right)$ conidia suspension was added to 96 -well plates as required. The plates were then incubated at $37^{\circ} \mathrm{C}$ for $3 \mathrm{~h}$ for adhesion of the conidia to the surface of the wells under static conditions. Subsequently, the growth suspension was replaced with pre-sterilised $250 \mu \mathrm{L}$ of RPMI $1640(\mathrm{pH}$ 7) medium containing GF:HPMCAS (chloroform solvate) and non solvated (prepared from acetone). The concentration of the formulations used in this study were equivalent to the known $\mathrm{MIC}_{90}$ of griseofulvin against $T$. rubrum (8 $\mu \mathrm{g} / \mathrm{mL}$ ) as demonstrated previously [18] were prepared following clinical and 
laboratory standards institute (CLSI) guidelines M38-A2 for fungal work. Biofilm of T. rubrum NCPF 935 was grown with and without the addition of the formulation and grown for a period of $96 \mathrm{~h}$ at $37^{\circ} \mathrm{C}$ prior to carrying out subsequent assays.

The concentration of GF:HPMCAS (solvated and non-solvated) used in this study are provided in Table 1 (supplementary material). The total concentration (w/v) of GF:HPMCAS used for the in vitro studies was based on $\mathrm{MIC}_{90}$ of GF as per published literature. DMSO when diluted in RPMI without the formulations did not exhibit any inhibitory effects on T. rubrum NCPF 953.

\subsection{Metabolic activity of $T$. rubrum NCPF 935 by XTT assay}

XTT assay was used based on the method described by Costa-Orlandi et al., 2014 with a few modifications [16]. The assay was used in this study to quantify the metabolic activity of the biofilm with and without the presence of the formulations. Stock solutions of XTT (2,3-bis (2-methoxy-4-nitro-5sulfophenyl)-5- [carbonyl (phenylamino)]-2H-tetrazolium hydroxide) and menadione were prepared, $1 \mathrm{mg} / \mathrm{mL}$ in PBS and $1 \mathrm{mM}$ ethanol respectively, and stored at $4{ }^{\circ} \mathrm{C}$. T. rubrum NCPF 935 biofilms were grown with and without the presence of the formulations that showed the highest biofilm inhibition. Samples were analysed at $3,8,24,48,72$ and $96 \mathrm{~h}$. To the samples, $50 \mu \mathrm{L}$ of XTT solution was added along with $4 \mu \mathrm{L}$ of menadione into the 96-well plates and incubated at $37^{\circ} \mathrm{C}$ for $3 \mathrm{~h}$. The resulting formation of formazan salts and colorimetric change were measured in a plate reader at $490 \mathrm{~nm}$ (FLUOstar Optima, BMG labtech, UK). RPMI 1640 medium, free of biofilm was used as a negative control. 


\subsection{Protease activity of $T$. rubrum NCPF 935}

Proteolytic activity of $T$. rubrum NCPF 935 was measured with and without the presence of formulations. The method used in this study was based on AlJanabi and Al-Hamadani, 2015 with a few modifications [19]. Briefly, T. rubrum NCPF 935 was grown in PDB (potato dextrose broth) (Sigma-Aldrich, UK) at $37^{\circ} \mathrm{C}$ in a rotary shaker at 4 RCF for 7 days. Casein from bovine milk $(1 \% \mathrm{w} / \mathrm{v})$ (Sigma-Aldrich, UK) was used to determine the proteolytic activity. Briefly, to $500 \mu \mathrm{L}$ of the casein substrate, $500 \mu \mathrm{L}$ of $T$. rubrum NCPF 935 filtrate treated with and without the formulations was added along with $100 \mu \mathrm{L}$ of $0.5 \mathrm{M}$ tris- $\mathrm{HCL}(\mathrm{pH} 8)$ in test tubes. The reaction was carried out at $40^{\circ} \mathrm{C}$ for $30 \mathrm{~min}$ and stopped by adding $2 \mathrm{~mL}$ of $0.67 \mathrm{M}$ trichloro acetic acid (TCA). The test tubes were allowed to rest at room temperature for $1 \mathrm{~h}$. The precipitate formed was removed by centrifugation at $5000 \mathrm{rpm}$ for $15 \mathrm{~min}$. The absorbance was then measured at $280 \mathrm{~nm}$ using a spectrophotometer against a blank where TCA was added prior to the incubation period. Unit of enzymatic activity was defined as the amount of enzyme that produced products by breaking down casein giving an absorbance of 0.1 at $280 \mathrm{~nm}$ under the experimental conditions. The following formula was used to calculate the activity of protease per $\mathrm{mg}$ of protein.

Protease activity $(\mathrm{U} / \mathrm{mL})=\mathrm{OD}(280 \mathrm{~nm}) /(0.01 \times$ Time $\times$ volume $)$ 


\subsection{Confocal laser scanning microscopy of T. rubrum NCPF 935 biofilm}

Biofilms of $T$. rubrum NCPF 935 were grown in 6-well tissue culture (Nunc) plates in RPMI- 1640 medium at $37^{\circ} \mathrm{C}$ for a period of $72 \mathrm{~h}$ with and without the addition of GF:HPMCAS (chloroform solvate) and non solvated (prepared from acetone). FUN-1 (Molecular probes, Invitrogen) was prepared according to manufactures instructions and was used to stain T. rubrum NCPF 935 biofilm as mentioned by Costa-Orlandi et al., 2014 with a few modifications [16]. Briefly, the biofilm was gently washed using $0.9 \%(\mathrm{v} / \mathrm{v})$ saline solution and treated with $300 \mu \mathrm{L}$ of FUN-1 fluorescent stain for 45 min and incubated at $37^{\circ} \mathrm{C}$ protected from light. After the incubation period, the excess stain was gently washed using HEPES buffer after which, the biofilms were visualised at $\sim 485 \mathrm{~nm}$ excitation/ $530 \mathrm{~nm}$ emission (green) using a Leica TSC SP2 confocal microscope (Heidelberg, Germany).

\subsection{Data analysis}

All experiments were conducted in triplicate unless mentioned otherwise. Statistical analysis was conducted using one-way ANOVA (Tukey's multiple comparision test). A $P$ value of $<0.05$ was considered to be significant. Data processing was performed using GraphPad Prism 7 for windows, GraphPad Software, La Jolla California, USA. 


\section{Results}

\subsection{Thermal analysis of GF solid dispersions}

Assessment of drug/polymer interaction was based on solid solubility, which was evaluated using melting point depression of corresponding physical mixtures (Fig 1). As can be seen in Fig. 1, GF-HPMCAS showed lowest depression of approximately $50{ }^{\circ} \mathrm{C}$. On the contrary there was minimal change in GF melting in silica and MCC dispersions signifying minimum impact on the crystallinity of GF. Due to degradation of PVP, measurement of GF melting in GF/PVP was not possible.

The solvated solid dispersions were also evaluated to determine any changes in the melting point (Fig 1). Formation of the GF solvate was evident in the DSC thermograms where an endothermic peak was clearly visible at approximately $97^{\circ} \mathrm{C}$ (Fig. 1). This peak corresponds to the melting of chloroform solvate crystals, which was confirmed via cooling cycle that showed disappearance of this endotherm. The endotherm seen was significant with a heat of fusion of approximately $84 \mathrm{~J} / \mathrm{g}$. Once the solvate melted, another endothermic event was seen at higher temperature attributed to the melt of the GF free crystals (non-solvated). The melting endotherm onset of GF was $218{ }^{\circ} \mathrm{C}$ for GF prepared in chloroform (solvated), 220 and $219^{\circ} \mathrm{C}$ for GF prepared in acetone and methanol (non-solvated), respectively. The heat of fusion of crystalline GF was $86 \mathrm{~J} / \mathrm{g}$ and reflected the presence of the chloroform solvate because the heat of fusion for GF re-crystalized in acetone and methanol was $115 \mathrm{~J} / \mathrm{g}$. This difference in heat of fusion reflects a mixture of crystalline structures, i.e. crystalline GF and crystalline GF- 
chloroform crystals. These results agreed with previous research on GF solvates where the melting of the solid-chloroform solvate occurred at $147^{\circ} \mathrm{C}$ [20]. The lower melting peak in our results is caused by the mixing with different polymers which affected the onset of the endotherm but not the extent of solvation. As can be seen in Fig. 1, the chloroform solvate peaks were observed in the temperature range $85-135{ }^{\circ} \mathrm{C}$. Differences in the melting onset seen among different excipients reflect variations in molecular interactions where HPMCAS dispersions showed lowest onset of solvate melting. Variation of the molar ratio of the solvent-GF were found previously to affect the onset of the melt as was shown for different solvates [21]. This possible variation was avoided via using similar ratio of the solvent throughout this study.

\subsection{Characterization of formed dispersions by XRPD}

The effect of the polymer on solvate formation was studied by analysing the crystalline patterns (Fig. 2). The evidence of the solvate form was confirmed by the observation of the peaks at $11.3^{\circ}, 15.3^{\circ}$ and $26.5^{\circ}$. . It was also clear that some of the peaks were less intense in the drug/polymer mixtures. This could indicate presence of the partially amorphous form formation because of the influence of the polymer.

The formation of the solvate was investigated further and the results can be seen in Fig. 2. New peaks were seen in the scan at $2 \theta$ of $11.2^{\circ}, 15.3^{\circ}, 17.5^{\circ}$, $22.5^{\circ}, 26.5^{\circ}$ while other peaks such as at $10.8^{\circ}, 28^{\circ}$ and $32.8^{\circ}$ diminished in 
the chloroform GF solvate. These results indicate that the GF chloroform solvate was formed. It was still possible to observe other peaks, which indicates that the formed solvate contributed only partially to the crystalline structure of GF. Changes from the chloroform solvate orthorhombic form to non-solvated tetragonal (depicted from the crystalline structures) were observed when temperature was increased from 30 to $200^{\circ} \mathrm{C}$ and the solvate disappeared completely at $120^{\circ} \mathrm{C}$. These results were similar for all drugpolymer combinations. The peaks positions, and in particular at $11.2^{\circ}$, were changing gradually and started to appear at $80^{\circ} \mathrm{C}$.

Fig. 2: (A) X-ray powder diffraction scans of 1:1 (GF : polymer) prepared in acetone, chloroform and methanol and $(B) X$-ray powder diffraction scans of 1:1 (GF:HPMCAS) chloroform solvate as a function of temperature showing polymorphic changes that start to occur $\sim 90{ }^{\circ} \mathrm{C}$. These changes result from the loss of the solvate structure at this temperature range.

\subsection{Hydrogen bonding analysis of polymer-GF interactions}

While the formation of GF solvates was reported previously for different solvents [22, 23], limited studies were carried out of solvated polymeric dispersions. Fig. 3 shows the FTIR spectra obtained from 1:1 GF-polymer solid dispersions in acetone, chloroform and methanol. The very broad peak at $1730-1750 \mathrm{~cm}^{-1}$ is associated with the carbonyl group stretching of HPMCAS. The peaks at $1705 \mathrm{~cm}^{-1}$ and $1657 \mathrm{~cm}^{-1}$ correspond to the stretching of the benzofuran and cyclohexene carbonyl groups in GF [24]. In the chloroform solid dispersion these peaks have shifted to $1694 \mathrm{~cm}^{-1}$ and 1666 $\mathrm{cm}^{-1}$ respectively, indicating the presence of hydrogen bonding between griseofulvin and chloroform. 
The peaks at $1615 \mathrm{~cm}^{-1}$ and $1585 \mathrm{~cm}^{-1}$ are typically associated with the $\mathrm{C}=\mathrm{C}$ stretch of the aromatic ring in griseofulvin. Interestingly, in the spectrum of the chloroform solid dispersion the small peak at $1599 \mathrm{~cm}^{-1}$ which represents the aromatic $\mathrm{C}=\mathrm{C}$ stretch in griseofulvin disappears. Its absence indicated significant polymorphic changes. The shifts were identical among different polymers, which indicated predominant impact of the solvent (chloroform) in the solvate formation.

\subsection{Effect of different polymers on the solubility of griseofulvin (GF)}

The saturation solubility of GF solid dispersions prepared in acetone, chloroform and methanol are shown in Fig. 4. The solubility of GF chloroform solvate was the lowest compared to that prepared from acetone or methanol (35, 45 and $70 \mu \mathrm{g} / \mathrm{mL}$, respectively). However, as shown in Fig. 4, incorporation of the polymers has increased the solubility of the chloroform dispersion compared to GF alone. The extent of this enhancement ranged from $31 \%$ for GF-MCC to $132.9 \%$ for GF-HPMCAS solid dispersions as measured after 72 hours. The solubility patterns in the initial 5 hours were largely similar to that of saturation solubility, with HPMCAS being the most soluble and MCC being the lowest in this ascending order: MCC < silica < PVP $<$ HPMCAS. 
The ability of HPMCAS to improve drug dissolution and prevent drug recrystallization was also evident. HPMCAS proved to be the most effective polymer to enhance solubility, with an increase up to $150 \%$. This was particularly evident in GF-HPMCAS chloroform solvate, which outperformed the GF-HPMCAS in acetone solid dispersion by as much as $73 \%$. Improvements in solubility were also evident in all other polymers, with the exception of silica, which solubility is almost similar to that in acetone dispersions.

\subsection{Antifungal activity of solvate dispersions}

Formation of a biofilm is essential in the pathogenicity of $T$. rubrum to cause chronic superficial fungal infections such as onychomycosis (fungal nail infections). In vitro growth of T. rubrum NCPF 935 and subsequent antifungal assays displayed a significant reduction in the biofilm production $(p<0.0001)$ after the exposure to GF:HPMCAS chloroform solvate and non-solvated GF:HPMCAS prepared from acetone when compared to the untreated control.. GF chloroform solvate was found to be more effective in antagonising the production of the biofilm compared to non-solvated GF prepared from 
acetone as shown in Fig. 5 (a, b, c). As both forms of GF showed significant reduction in biofilm compared to the untreated control, this in turn reflects the impact of preparing the drug as a solid dispersion and the possible antagonistic activity of HPMCAS towards the negating the pathogenicity of $T$. rubrum. The antifungal activity of solvated (chloroform) and non- solvated (acetone) GF against $T$. rubrum NCPF 935 is clearly observed with the XTT assay wherein a significant reduction in metabolic activity was observed after 8h (Fig 5). This decreased metabolic activity was observed for the entire duration of the experiment starting $8 \mathrm{~h}$ at time point. Data obtained from the XTT assay correspond to the reduction of the biofilm with the treatment due to lowered activity of $T$. rubrum NCPF 935 and its ability to produce biofilm. As shown in Fig 5, the GF chloroform solvate and non-solvated GF prepared from acetone showed a significant reduction in protease activity. This reflects reduced keratinolytic activity, which is important to note as one of the primary virulence factors of dermatophytes.

Formation of hyphae is a prerequisite for fungal attachment to a substratum and formation of the biofilm. Assessing the effect of GF formulations on $T$. rubrum NCPF 935 hyphae and biofilm development by Confocal laser scanning microscopy showed a change in morphology of $T$. rubrum NCPF 935 hyphae (density, thickness) as well as absence of conidia with treatment after a period of $72 \mathrm{~h}$. Treatments therefore showed significant antagonism towards hyphal formation and subsequent propagation and spread of the 
fungi. Similarly, absent conidia signify the reduced ability of $T$. rubrum NCPF 935 to sporulate, thereby negating the spread of the infection.

The chloroform solvate of GF-HPMCAS was found to have a higher impact on inhibiting the biofilm development of $T$. rubrum NCPF 935. This could be clearly seen in Fig 5, as thicker hyphae but not as dense were observed in GF-HPMCAS dispersions (non-solvated). On the contrary, significant reduction in the thickness and density as thin hyphae could be seen in the GF-HPMCAS dispersions (solvated). Overall, a lack of conidia in T. rubrum NCPF 935 was evident when treated with the GF dispersions.

\subsection{Analysis of solid and liquid state interactions using solid and liquid} state NMR

Solvated GF-HPMCAS dispersion showed higher antifungal activity when compared to non-solvated form. To fully understand the mechanism of this enhancement, it is important to find whether the crucial interactions, which were established in the liquid state, are also retained in the solid state. Nuclear magnetic resonance (NMR) is particularly suited to tackle such a 
problem because its offer direct inside into molecular structure in the solution (liquid state NMR) and solid (solid-state NMR) and its able to provide detailed information on a very subtle interactions, including hydrogen bonds.

To be able to correctly compare and analyse solution and solid-state NMR results, the full assignment of all signals in ${ }^{13} \mathrm{C}$ NMR spectra of GF was required (Fig. 6 and Table 1). The assignment of the signals $i{ }^{13} \mathrm{C} N M R$ spectrum of GF was found to be in good agreement with previously published data [25] as presented in Fig. $6 \mathrm{~A}$ and Table $1 .{ }^{13} \mathrm{C}$ solid-state NMR spectrum of pure GF showed the presence of additional signals (195 ppm and new signal between 91 and 90 ppm), which could not be attributed to spinning sidebands. This strongly suggests the coexistence of different isomers, possibly with distinctive patterns of hydrogen bonding in the crystalline dispersion as observed before [26]. This is supported by the observation that resonances from the carbons $\mathrm{C} 5, \mathrm{C} 2$ which should be particularly sensitive to hydrogen bonding are present around 195 ppm and in the region between 95 and 90 ppm.

The ${ }^{13} \mathrm{C}$ solid-state NMR spectra of GF, HPMCAS and GF and HPMCAS dispersions are compared in Fig. 6B. There was no significant difference between the spectra of physical mixture of GF and HPMCAS and a sum of the separate GF and HPMCAS spectra (Fig. 6B). This suggests negligible interaction in the physical mixture regardless of any conformational differences in the GF isomers. 
Fig. 6B (ii-v) compares ${ }^{13} \mathrm{C}$ solid-state NMR spectra of GF:HPMCAS dispersions prepared from chloroform, acetone and methanol with the spectrum of the physical mixture. For dispersions prepared from methanol, no differences were observed in the chemical shifts between the physical mixture and methanol dispersion indicating minimum impact of methanol on the formation of the dispersion. On the other hand, the dispersion prepared using acetone showed significant broadening of the signals attributed to GF with mostly unchanged HPMCAS signals. For example, peaks at 97.9 and 94.5 ppm have merged into one broad structure at $96 \mathrm{ppm}$. These changes indicate the formation of amorphous GF dispersion, which agreed with the XRPD data shown above.

When comparing the chemical shifts in the spectra of the dispersion prepared from chloroform, significant changes were observed especially for $\mathrm{C} 14$ and C21 carbons (Table 1, Fig. 6A). The downfield shift in $\delta_{\mathrm{C} 14}\left(\delta_{\text {solid }}<\delta_{\text {solution }}<\right.$ $\delta_{\text {complex})}$ suggests that stronger hydrogen bonding in the chloroform complex compared to chloroform solution. In general, there were significant differences in the carbon chemical shift between solution, complex dispersion and crystalline samples. This is consistent with the highly constrained crystalline structure, compared with the solution where mobility of the methyl groups is less restricted. Furthermore, general analysis of the 
chemical shifts, suggests that the structure of the chloroform complexdispersion was closest to that observed in the chloroform solution (Table 1).

\section{Discussion}

GF was shown before to have weak activity against T. rubrum isolates [27]. However, in our work, the metabolic and protease activity of T. rubrum NCPF 935 with and without the presence of GF:HPMCAS chloroform solvates showed significant reduction compared to the untreated control after $24 \mathrm{~h}$ period. This was clearly visible in confocal laser scanning microscopy which showed disappearance of the hyphae compared to Control and GF:HPMCAS (non solvated). Chloroform extracts were shown before to have low activity against $T$. rubrum fungal strains [28]. Therefore, results shown demonstrate novel role for the polymeric dispersion in augmenting the activity of the antifungal drug GF.

HPMCAS solvate dispersions were more stable when compared with other polymers, which may explain the observed synergistic effect. As can be seen in Figure 7, dynamic vapour sorption data showed recrystallization of all solvate dispersions when the relative humidity was increased to around $70 \%$. The loss in mass was a sign of loss of bound and non-bound water (or solvate). No recrystallization (or solvate loss) could be observed in HPMCAS solvate dispersions upon sorption and desorption cycles which indicates that the solvates maintained their structures when exposed to water. 
Hydrogen bonding between the hydrogen in chloroform molecule and the carbonyl on GF explains the basis for GF chloroform solvate formation, which was clearly visible in the FTIR analysis. This ability to form hydrogen bonding is based on the fact that chloroform acts as Lewis acid with the potential to form hydrogen bonds with corresponding bases [29, 30]. When different polymers were incorporated, the GF solvate was affected. For example, HPMCAS resulted in the disruption of the solvate crystalline lattice likely by formed hydrogen bonding as was shown in ${ }^{13} \mathrm{C}$ solid-state NMR spectra. Polymorphic changes were confirmed using XRPD where data showed despite that part of GF existed as amorphous; the main polymorphic structure was the solvate form. This was clear in Fig 2 where the increase in the temperature to $80^{\circ} \mathrm{C}$ was associated with the disappearance of the solvate and appearance of form I GF polymorph. The extensive hydrogen bonding for GF-HPMCAS chloroform solvate has been shown to enhance the solubility of GF. This trend has also been seen for PVP where improvement in solubility could also be observed when compared with the solubility of silica and MCC dispersions.

This suggests that solvates, which were formed in the liquid state, maintained their structures in the solid state. Such behaviour was reported previously where lithium solvates were shown to remain stable in solutions of aprotic solvents and affected the properties of those solutions [31]. Similarly, it is possible that solvates formed from chloroform maintained their molecular 
structure and assisted in enhancing solubility via $\mathrm{Cl} . . . \mathrm{Cl}$ interactions together with the formed hydrogen bonds with GF and HPMCAS. This could be possible reason for the increased antifungal activity of GF-HPMCAS solvate, which was 2-5 times stronger when compared with the activity of GF alone. Synergistic effect was also caused by prevention of recrystallization and solvate expulsion as evident in Fig. 7, which could explain the disruptive effect of GF-HPMCAS on biofilms..

\section{Conclusions}

The results of this work showed that the griseofulvin solvate had a significantly higher antifungal activity against dermatophytes compared to non-solvated form. This synergistic effect was achieved by carefully planned crystal engineering in which the drug properties were changed via dispersing it into a matrix. Solvated crystals of griseofulvin embedded within the HPMCAS matrix showed higher dissolution properties with evidence of hydrogen bonding. The most significant impact of these distinctive molecular interactions present in the solvate form was observed in the antimicrobial assay where the fungal threads dramatically diminished when treated with the chloroform solvate form of griseofulvin. Solution and solid-state NMR experiments demonstrated that solvent molecules play important role in the formation of very specific microstructure of the GF solid dispersions. In particular, the interaction between GF and chloroform were found to be similar in solution and solvate, which could be responsible for the unique properties 
of the solvate. Our findings further signify the role of precise crystal engineering in preparing highly potent versions of griseofulvin.

\section{Declaration of Interest}

The authors report no conflict of interests.

\section{Acknowledgements}

The authors would like to thank the Chemical Analysis Facility (CAF) at the University of Reading for providing essential access to instruments used in this study. We would like to thank Dr Kenneth Shankland for his help and advice with the temperature controlled x-ray powder diffraction experiments.

\section{References}

[1] R.M. Donlan, Biofilms: Microbial life on surfaces, Emerg Infect Dis, 8 (2002) 881-890.

[2] K.F. Mitchell, R. Zarnowski, D.R. Andes, The Extracellular Matrix of Fungal Biofilms, Advances in experimental medicine and biology, 931 (2016) 21-35.

[3] N. Hoiby, T. Bjarnsholt, C. Moser, G.L. Bassi, T. Coenye, G. Donelli, L. HallStoodley, V. Hola, C. Imbert, K. Kirketerp-Moller, D. Lebeaux, A. Oliver, A.J. Ullmann, C. Williams, E.S.G.f. Biofilms, Z. Consulting External Expert Werner, ESCMID guideline for the diagnosis and treatment of biofilm infections 2014, Clinical microbiology and infection : the official publication of the European Society of Clinical Microbiology and Infectious Diseases, 21 Suppl 1 (2015) S125.

[4] N. Toukabri, S. Corpologno, M.E. Bougnoux, D. El Euch, N. Sadfi-Zouaoui, G. Simonetti, In vitro biofilms and antifungal susceptibility of dermatophyte and non-dermatophyte moulds involved in foot mycosis, Mycoses, 61 (2018) 79-87.

[5] A.K. Sahoo, R. Mahajan, Management of tinea corporis, tinea cruris, and tinea pedis: A comprehensive review, Indian dermatology online journal, 7 (2016) 77 86.

[6] A.B. Wingfield, A.C. Fernandez-Obregon, F.S. Wignall, D.L. Greer, Treatment of tinea imbricata: a randomized clinical trial using griseofulvin, terbinafine, itraconazole and fluconazole, The British journal of dermatology, 150 (2004) 119-126. 
[7] A.K. Gupta, J.V. Williams, M. Zaman, J. Singh, In vitro pharmacodynamic characteristics of griseofulvin against dermatophyte isolates of Trichophyton tonsurans from tinea capitis patients, Medical mycology, 47 (2009) 796-801.

[8] S. Stegemann, F. Leveiller, D. Franchi, H. de Jong, H. Linden, When poor solubility becomes an issue: from early stage to proof of concept, Eur J Pharm Sci, 31 (2007) 249-261.

[9] S.S. Hate, S.M. Reutzel-Edens, L.S. Taylor, Insight into Amorphous Solid Dispersion Performance by Coupled Dissolution and Membrane Mass Transfer Measurements, Mol Pharm, 16 (2019) 448-461.

[10] H.C. Arca, L.I. Mosquera-Giraldo, J.M. Pereira, N. Sriranganathan, L.S. Taylor, K.J. Edgar, Rifampin Stability and Solution Concentration Enhancement Through Amorphous Solid Dispersion in Cellulose omega-Carboxyalkanoate Matrices, J Pharm Sci, 107 (2018) 127-138.

[11] A.M. Healy, Z.A. Worku, D. Kumar, A.M. Madi, Pharmaceutical solvates, hydrates and amorphous forms: A special emphasis on cocrystals, Adv Drug Deliv Rev, 117 (2017) 25-46.

[12] N. Hisada, R. Takano, N. Takata, K. Shiraki, T. Ueto, S. Tanida, M. Kataoka, S. Yamashita, Characterizing the dissolution profiles of supersaturable salts, cocrystals, and solvates to enhance in vivo oral absorption, Eur J Pharm Biopharm, 103 (2016) 192-199.

[13] G. Bolla, A. Nangia, Pharmaceutical cocrystals: walking the talk, Chemical communications, 52 (2016) 8342-8360.

[14] J. Li, K. Zhang, L. Ruan, S.F. Chin, N. Wickramasinghe, H. Liu, V. Ravikumar, J. Ren, H. Duan, L. Yang, M.B. Chan-Park, Block Copolymer Nanoparticles Remove Biofilms of Drug-Resistant Gram-Positive Bacteria by Nanoscale Bacterial Debridement, Nano letters, 18 (2018) 4180-4187.

[15] R.S.N. Brilhante, E.E.M. Correia, G.M.M. Guedes, V.S. Pereira, J.S. Oliveira, S.P. Bandeira, L.P. Alencar, A.R.C. Andrade, D. Castelo-Branco, R.A. Cordeiro, A.Q. Pinheiro, L.J.Q. Chaves, W.A. Pereira Neto, J.J.C. Sidrim, M.F.G. Rocha, Quantitative and structural analyses of the in vitro and ex vivo biofilm-forming ability of dermatophytes, J Med Microbiol, 66 (2017) 1045-1052.

[16] C.B. Costa-Orlandi, J.C. Sardi, C.T. Santos, A.M. Fusco-Almeida, M.J. MendesGiannini, In vitro characterization of Trichophyton rubrum and $\mathrm{T}$. mentagrophytes biofilms, Biofouling, 30 (2014) 719-727.

[17] R.M. dos Santos, M.V. Dias-Souza, Effectiveness of five antidandruff cosmetic formulations against planktonic cells and biofilms of dermatophytes, Saudi Journal of Biological Sciences, 24 (2017) 331-337.

[18] Y. Dabas, I. Xess, G. Singh, M. Pandey, S. Meena, Molecular Identification and Antifungal Susceptibility Patterns of Clinical Dermatophytes Following CLSI and EUCAST Guidelines, J Fungi (Basel), 3 (2017) 17.

[19] S.K. Kadhim, J.K. Al-Janabi, A.H. Al-Hamadani, In vitro, determination of optimal conditions of growth and proteolytic activity of clinical isolates of Trichophyton rubrum, Journal of Contemporary Medical Sciences, (2015) 919\%V 11.

[20] K. Sekiguchi, E. Suzuki, Y. Tsuda, Y. Morita, Thermal Analysis of GriseofulvinChloroform System by High Pressure Differential Scanning Calorimetry, Chem Pharm Bull (Tokyo), 31 (1983) 2139-2141.

[21] S. Aitipamula, P.S. Chow, R.B. Tan, Solvates of the antifungal drug griseofulvin: structural, thermochemical and conformational analysis, Acta 
crystallographica Section B, Structural science, crystal engineering and materials, 70 (2014) 54-62.

[22] K.C. Cheng, E. Shefter, T. Srikrishnan, Crystal structure analysis of the desolvation of the chloroform solvate of griseofulvin, International Journal of Pharmaceutics, 2 (1979) 81-89.

[23] B.A. Bolton, P.N. Prasad, Laser Raman investigation of pharmaceutical solids: griseofulvin and its solvates, J Pharm Sci, 70 (1981) 789-793.

[24] R. Nair, N. Nyamweya, S. Gonen, L.J. Martinez-Miranda, S.W. Hoag, Influence of various drugs on the glass transition temperature of poly(vinylpyrrolidone): a thermodynamic and spectroscopic investigation, Int J Pharm, 225 (2001) 83-96.

[25] S.G. Levine, R.E. Hicks, H.E. Gottlieb, E. Wenkert, Carbon-13 nuclear magnetic resonance spectroscopy of naturally occurring substances. XXX. Griseofulvin, The Journal of organic chemistry, 40 (1975) 2540-2542.

[26] A. Mahieu, J.F. Willart, E. Dudognon, M.D. Eddleston, W. Jones, F. Danede, M. Descamps, On the polymorphism of griseofulvin: identification of two additional polymorphs, J Pharm Sci, 102 (2013) 462-468.

[27] D.A. Santos, J.S. Hamdan, In vitro activities of four antifungal drugs against Trichophyton rubrum isolates exhibiting resistance to fluconazole, Mycoses, 50 (2007) 286-289.

[28] D. Rani, P.B. Khare, P.K. Dantu, In Vitro Antibacterial and Antifungal Properties of Aqueous and Non-Aqueous Frond Extracts of Psilotum nudum, Nephrolepis biserrata and Nephrolepis cordifolia, Indian journal of pharmaceutical sciences, 72 (2010) 818-822.

[29] A.V. Lesikar, Effect of association complexes on the glass transition in organic halide mixtures, The Journal of Physical Chemistry, 80 (1976) 10051011.

[30] F. Ito, Matrix-isolation infrared studies of 1:1 molecular complexes containing chloroform $(\mathrm{CHCl} 3)$ and Lewis bases: seamless transition from blueshifted to red-shifted hydrogen bonds, J Chem Phys, 137 (2012) 014505.

[31] D. Brouillette, D.E. Irish, N.J. Taylor, G. Perron, M. Odziemkowski, J.E. Desnoyers, Stable solvates in solution of lithium bis(trifluoromethylsulfone)imide in glymes and other aprotic solvents: Phase diagrams, crystallography and Raman spectroscopy, Physical Chemistry Chemical Physics, 4 (2002) 6063-6071. 

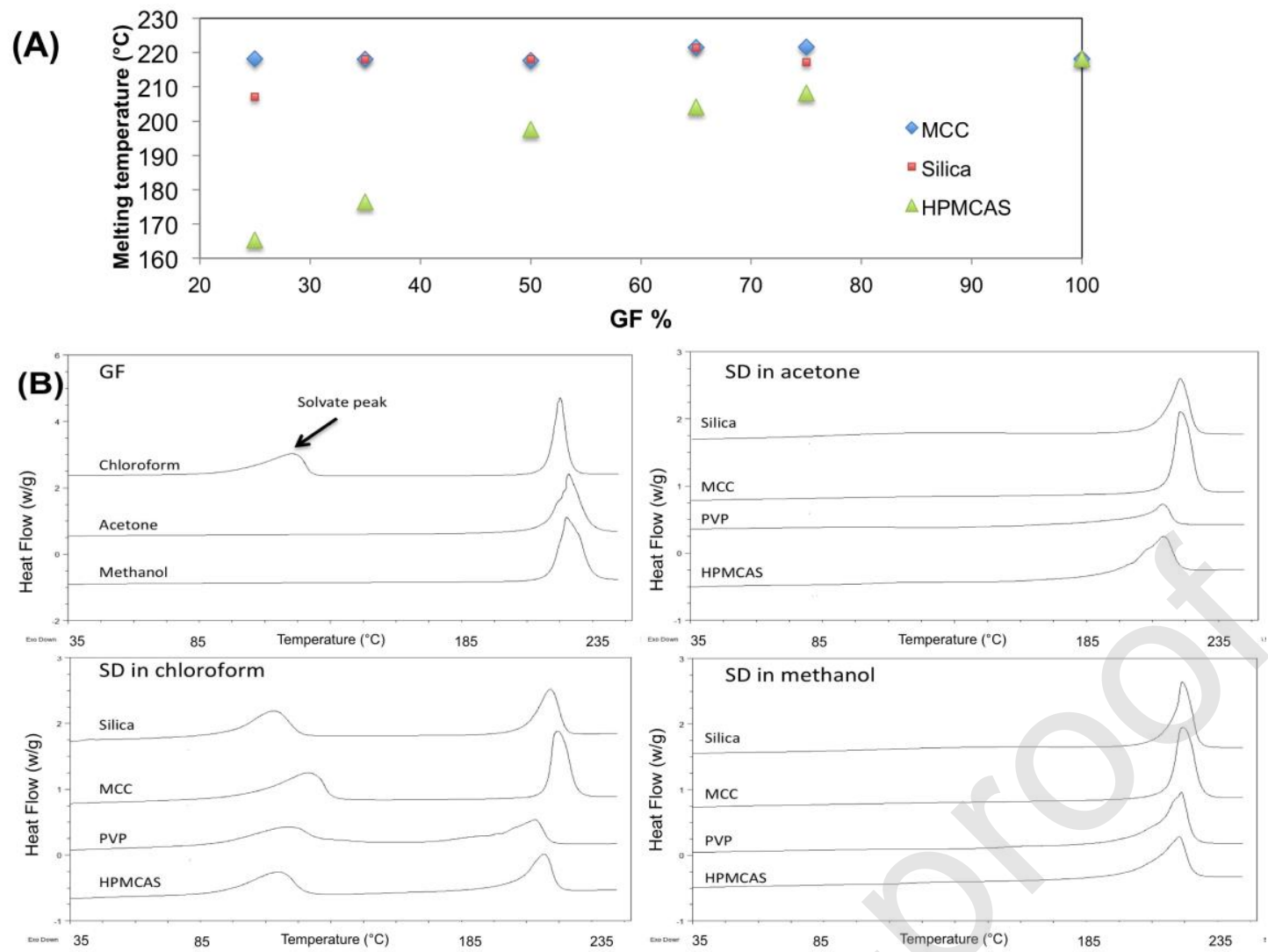

Fig. 1: Thermograms of GF mixtures with polymers $(A)$ showing the melting point of GF in physical mixtures with the polymers, and (B) showing thermograms of solid dispersions prepared in chloroform, methanol, acetone using a weight ratio of 1:1. The chloroform solvate peaks can be seen in the temperature range $85-135^{\circ} \mathrm{C}$.

(A)
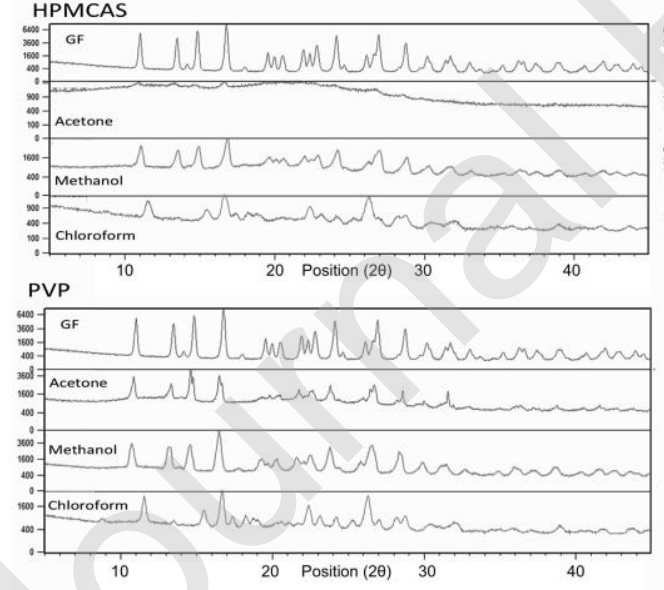
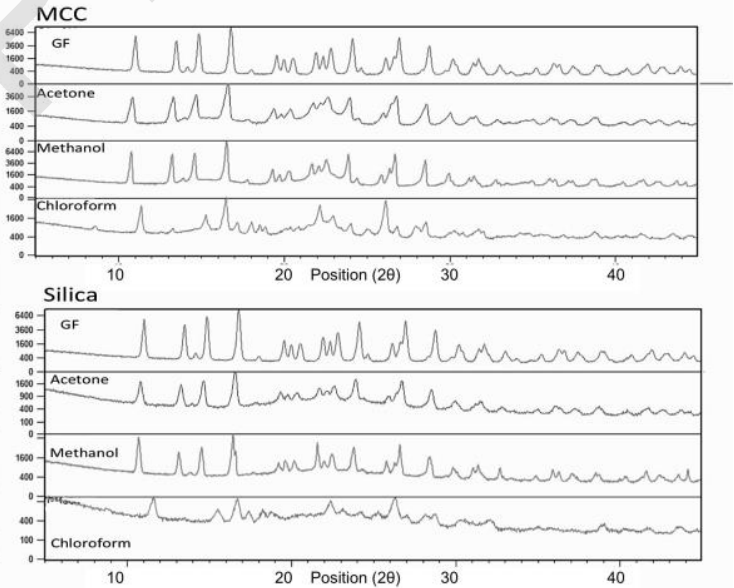

(B)

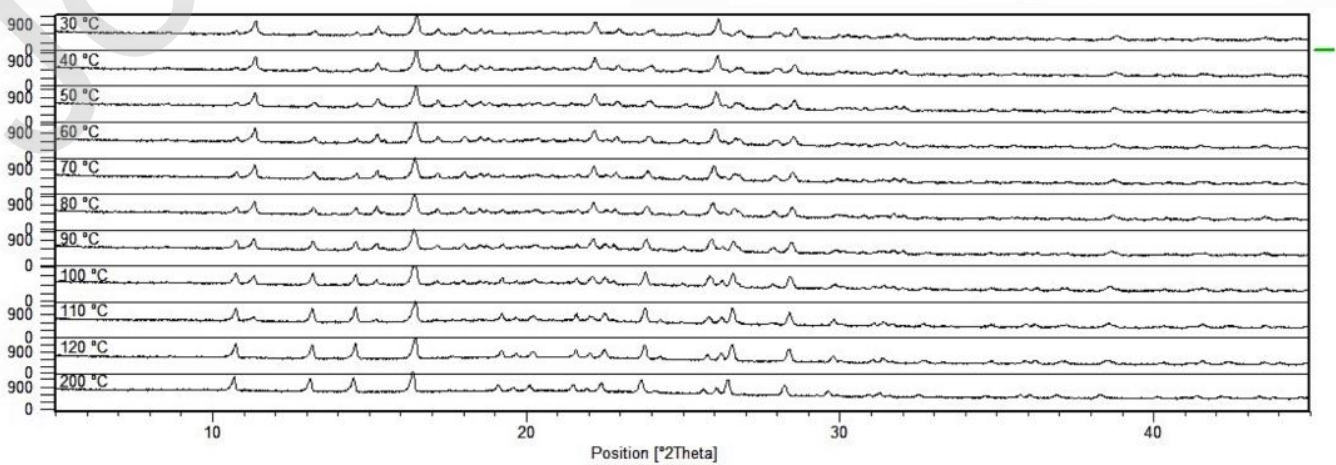


Fig. 2: (A) X-ray powder diffraction scans of 1:1 (GF : polymer) prepared in acetone, chloroform and methanol and $(B) X$-ray powder diffraction scans of 1:1 (GF:HPMCAS) chloroform solvate as a function of temperature showing polymorphic changes that start to occur $\sim 90{ }^{\circ} \mathrm{C}$. These changes result from the loss of the solvate structure at this temperature range.

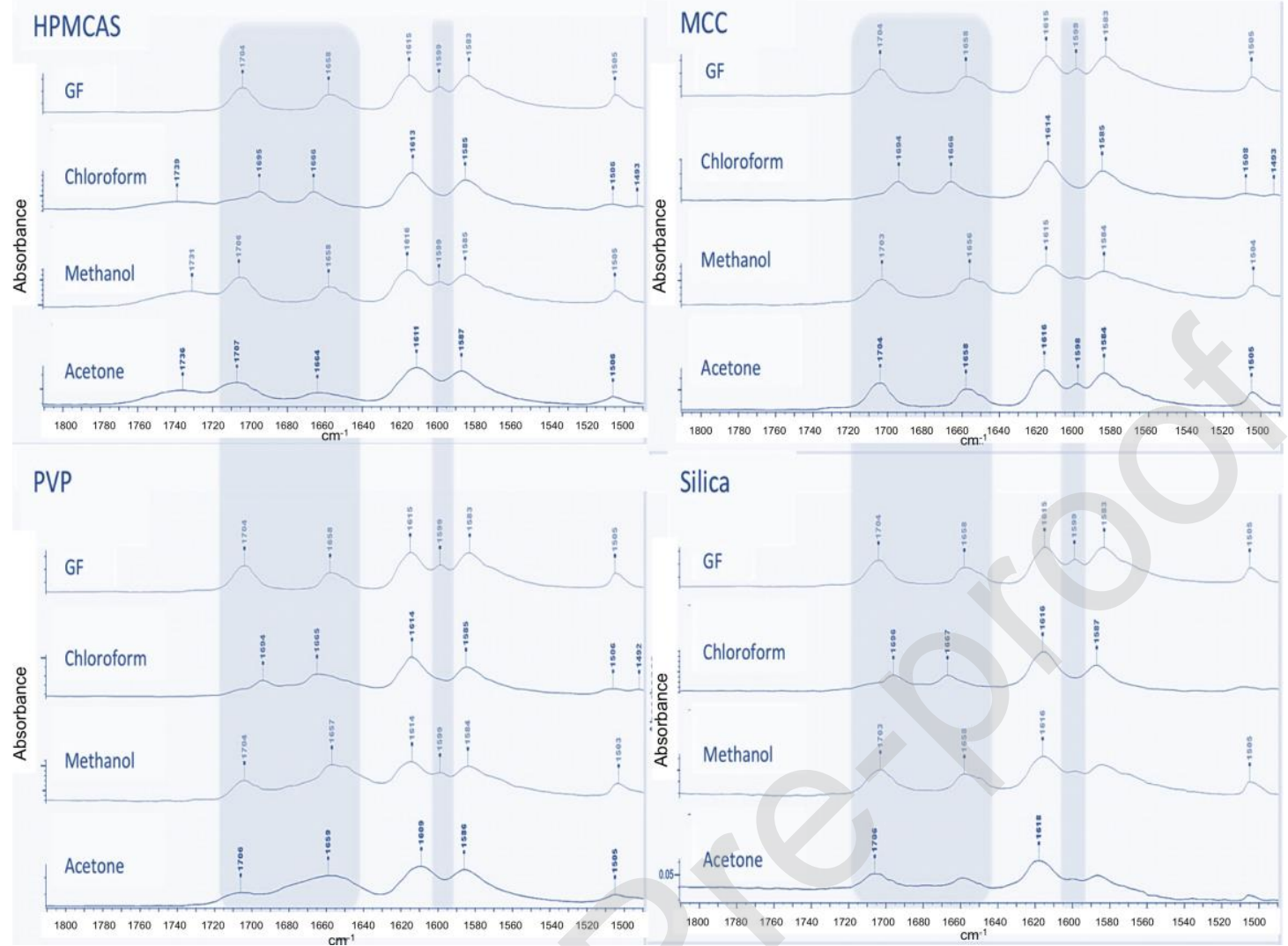

Fig. 3: FTIR scans of 1:1 (GF : polymer) prepared in acetone, chloroform and methanol showing significant changes in the chloroform solvates as reflected in the stretching vibrations highlighted by the shaded bars. 

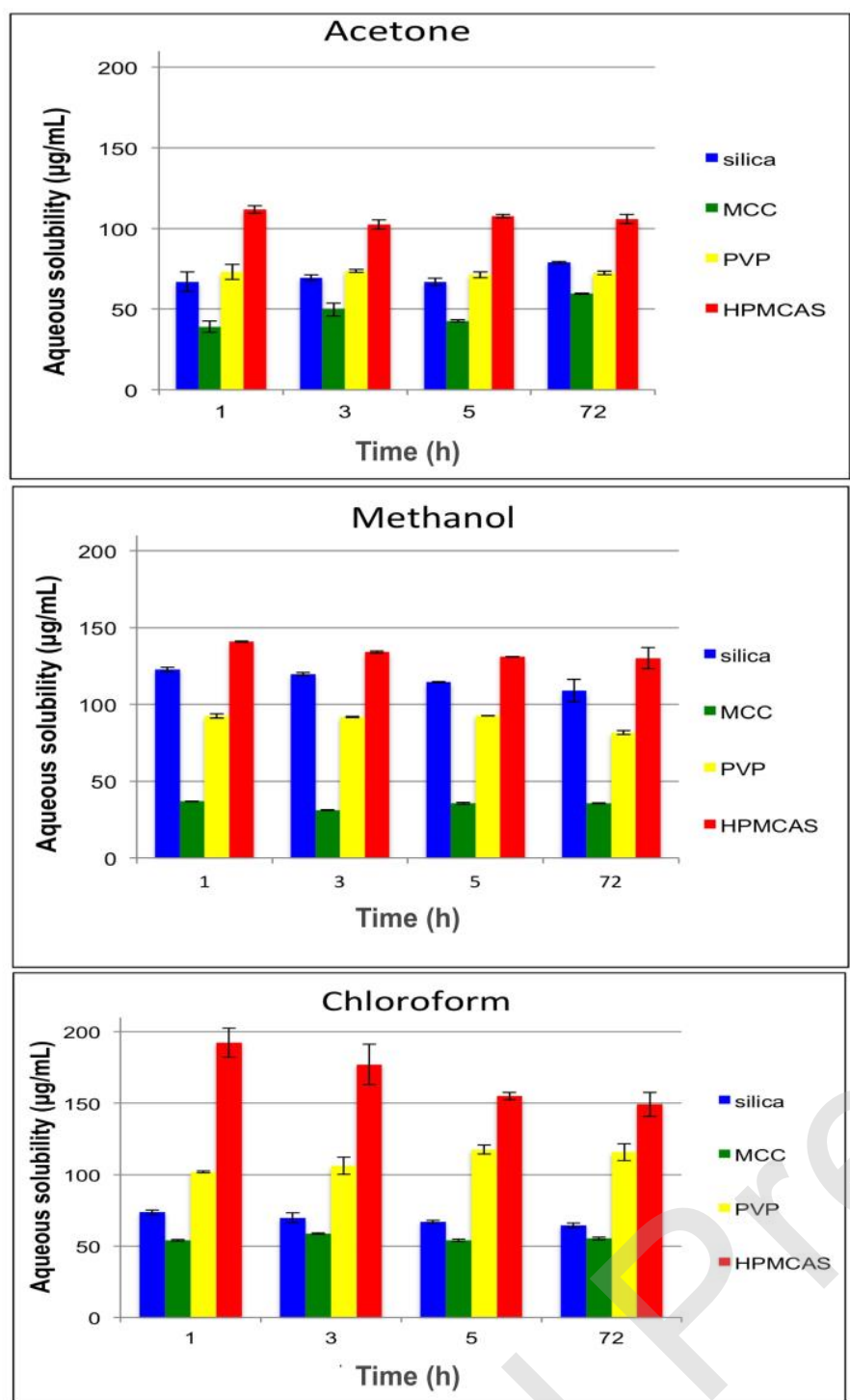

Fig. 4: saturated solubility measurements showing solubility of pure GF crystallized from different solvents and GF : polymer (1:1) prepared using different solvents. Measurements were performed in a phosphate buffer ( $p H=6.8$ ), $t=72$ hours, $n=3$. 

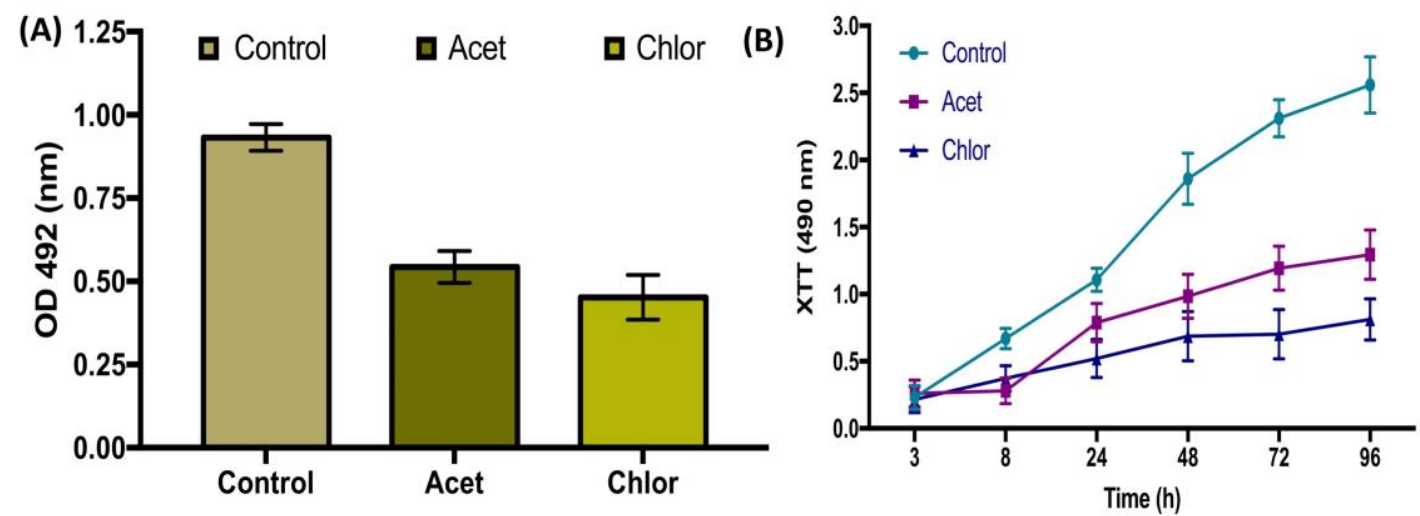

(c)

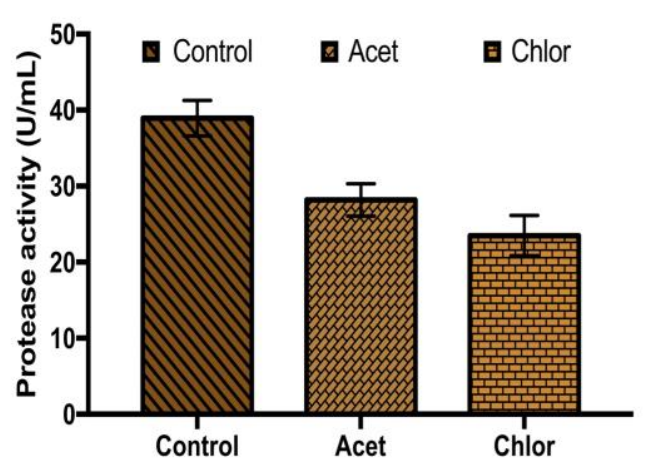

(D)
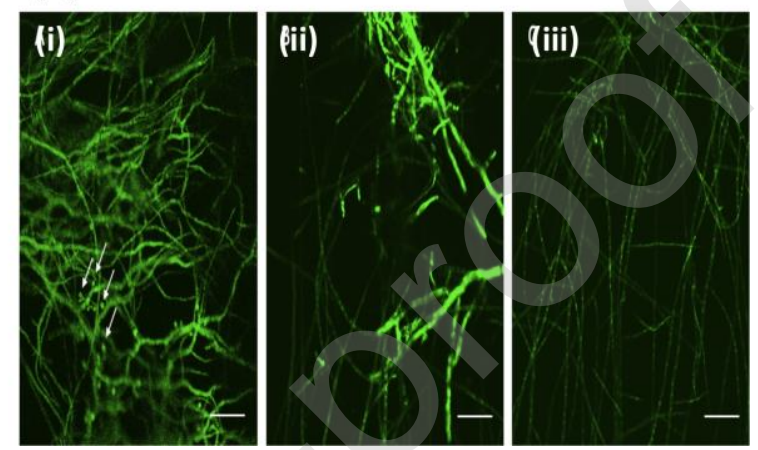

Fig. 5: (A) Quantification of biofilm formed by T. rubrum NCPF 935 by safranin staining. Compared to the control, the use solvated and non solvated GF:HPMCAS dispersions showed a significant reduction in biofilm content ( $p<$ 0.0001), (B) Metabolic activity of T. rubrum NCPF 935 with and without the presence of formulations GF:HPMCAS (chloroform solvate) and GF:HPMCAS non solvated (prepared from acetone) showed a significant reduction compared to the untreated control after $24 h$ period $96 h(n=3),(C)$ Quantification of protease activity by $T$. rubrum NCPF 935 showing significant reduction $(\mathrm{p}<0.0001)$ in protease activity $(\mathrm{n}=5)$ and $(D)$ confocal laser scanning microscopy images of T. rubrum NCPF 935 with and without treatment after $72 h$ (magnification at 630x). i) Control T. rubrum growth shows conidia (white arrows) and dense hyphae. ii) Treatment with GF:HPMCAS non solvated (prepared from acetone) showed thicker hyphae but not as dense. iii) Treatment with GF:HPMCAS (chloroform solvate) showed thin hyphae compared to Control and GF:HPMCAS non solvated (prepared from acetone). 


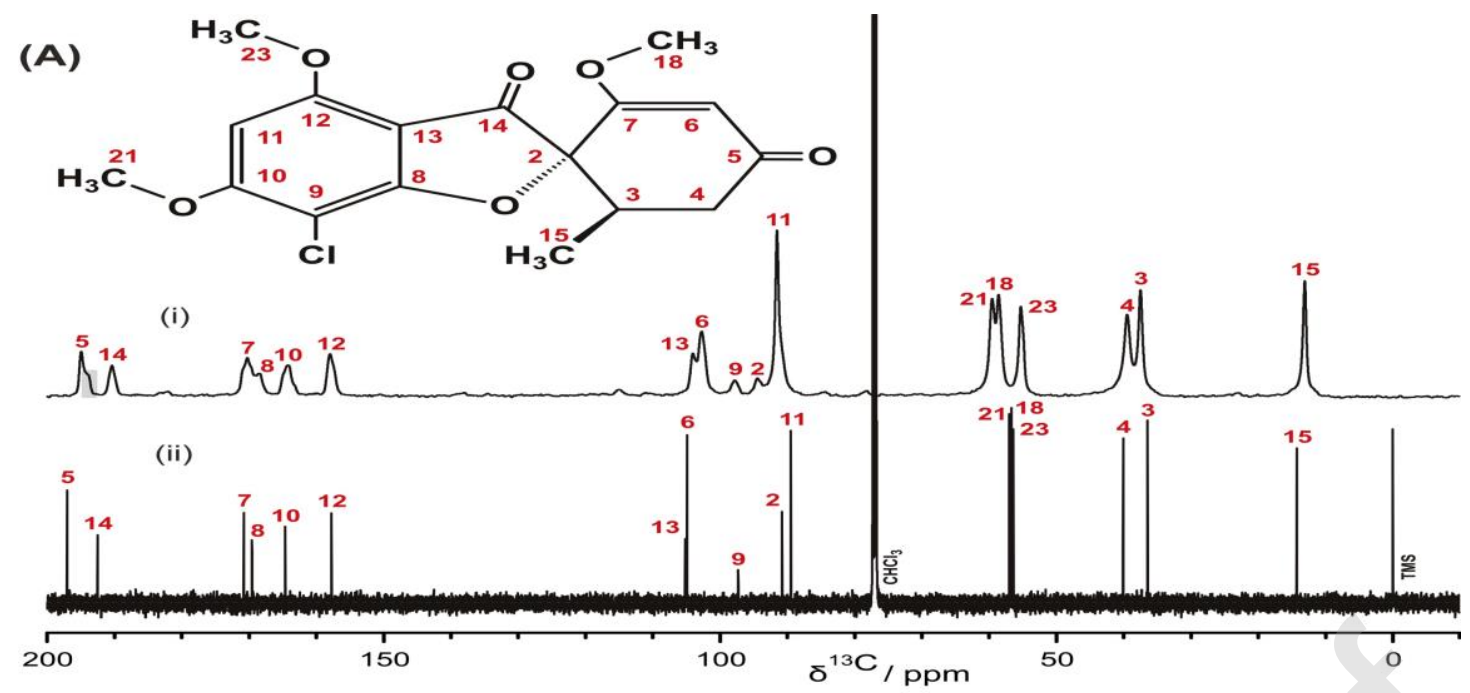

(B)
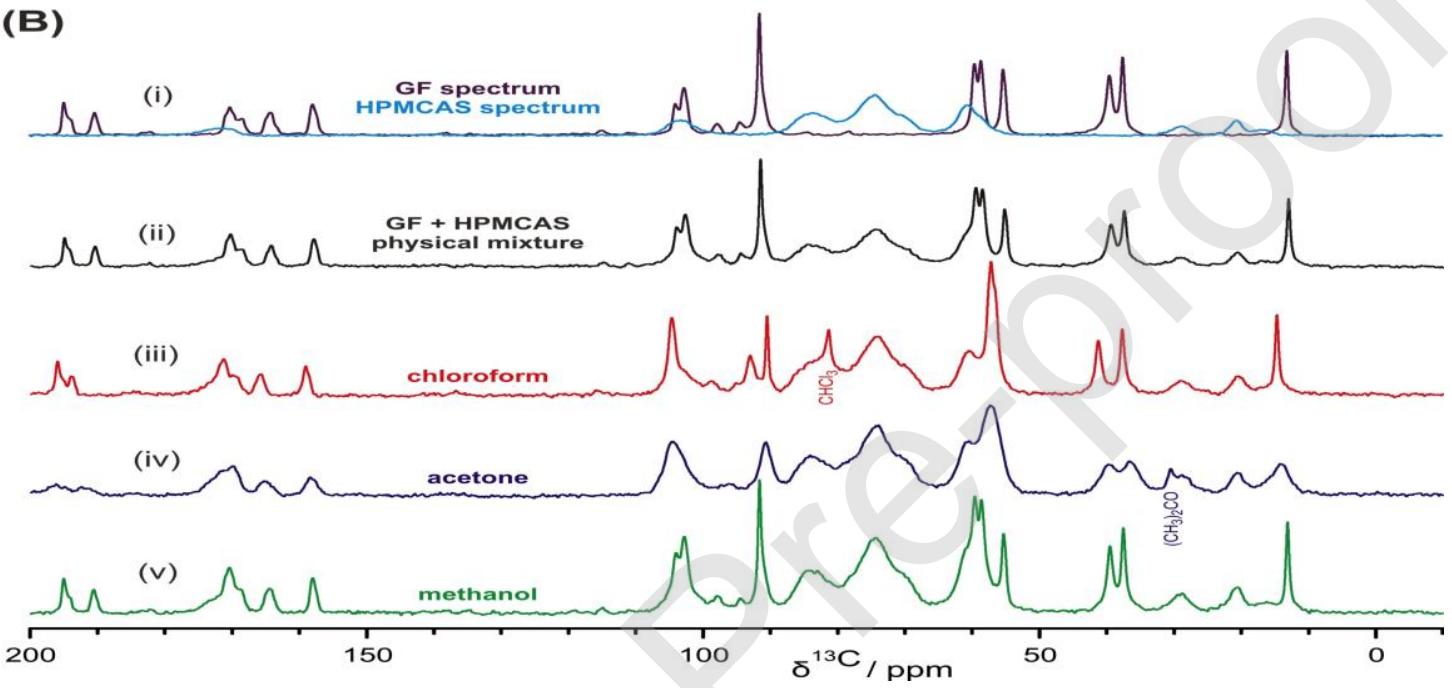

Fig. 6: (A) Molecular structure of GF and the assignment of the carbon atoms to the resonances recorded in (ii) solid-state and (i) solution ${ }^{13} \mathrm{C}$ NMR spectra. (B) Comparison of ${ }^{13} \mathrm{C}$ solid-state NMR spectra of (i) GF and HPMCAS, (ii) physical mixture of GF and HPMCAS, and GF:HPMCAS dispersions prepared from (iii) chloroform, (iv) acetone and (v) methanol. 

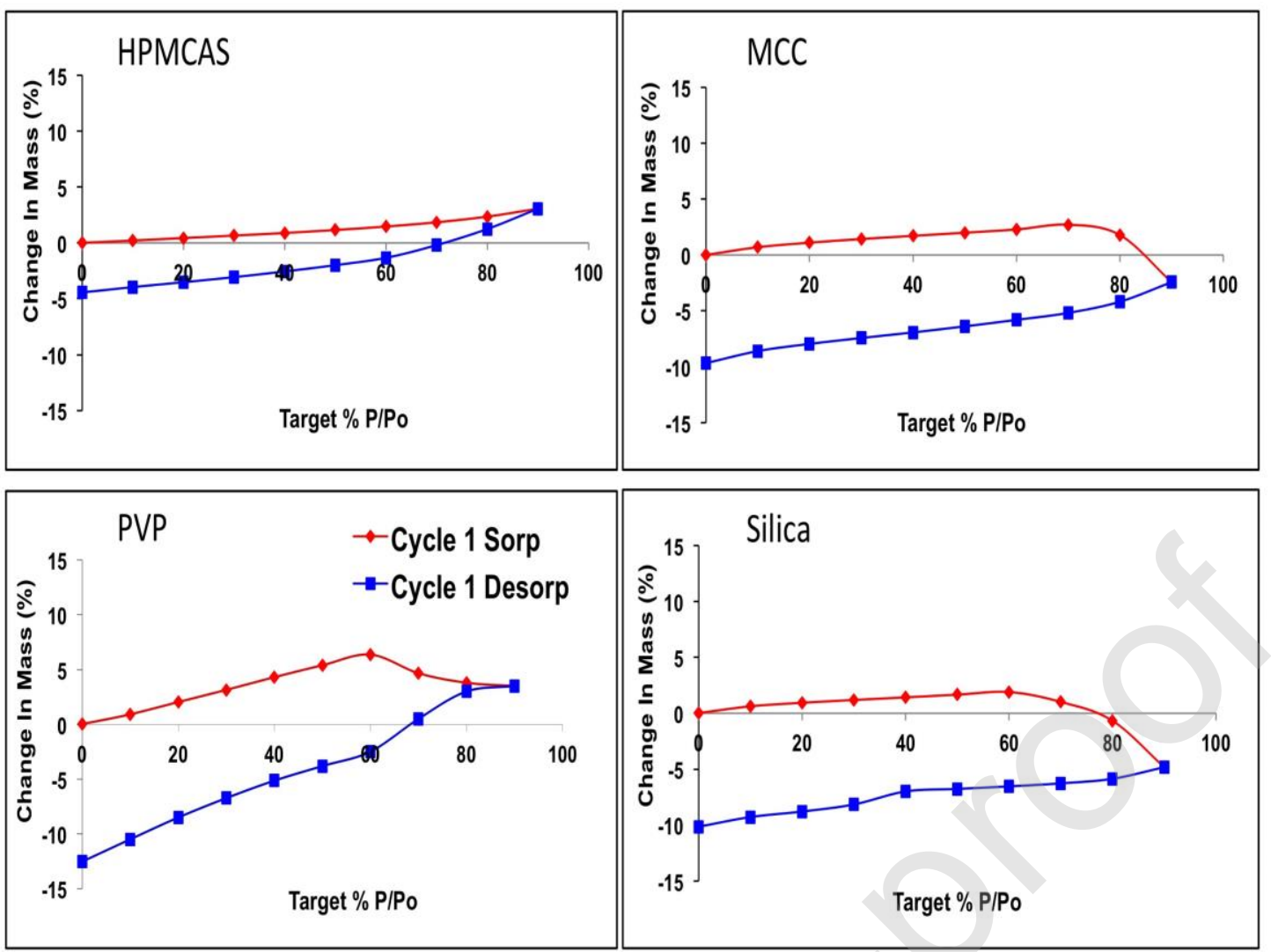

Fig. 7: Dynamic vapur sorption isotherms for GF solid dispersions prepared using chloroform.

Table 1: A summary of NMR chemical shifts as measured for GF:HPMCAS (1:1) using ${ }^{13} \mathrm{C}$ CPMAS and ${ }^{13} \mathrm{C}$ liquid NMR and their assignment to the molecular structure of GF (see Fig. 6A for full numbering of carbon atoms).

\begin{tabular}{|r|c|c|c|c|}
\hline \multirow{2}{*}{ Atom } & \multicolumn{4}{|c|}{${ }^{13}$ C chemical shift / ppm } \\
\cline { 2 - 3 } & \multicolumn{2}{|c|}{ solid state NMR experiment } & \multirow{2}{*}{$\begin{array}{c}\text { difference } \\
\text { between } \\
\text { physical mixture } \\
\text { and dispersion }\end{array}$} & $\begin{array}{c}\text { solution NMR } \\
\text { experiment }\end{array}$ \\
\cline { 2 - 3 } & physical mixture & dispersion & Chloroform \\
\hline C2 & $90.7^{\text {sh }}$ or 94.6 & 90.7 or 93.2 & - & 90.78 \\
\hline C3 & 37.5 & 37.8 & -0.3 & 36.44 \\
\hline C4 & 39.5 & 41.3 & -1.8 & 40.06 \\
\hline C5 & 195.0 & 195.9 & -0.9 & 197.04 \\
\hline C6 & 102.8 & 104.7 & -1.9 & 104.9 \\
\hline C7 & 168.5 & 170.0 & -1.5 & 170.78 \\
\hline C8 & 170.3 & 171.3 & -1 & 169.55 \\
\hline C9 & 97.8 & 98.9 & -1.1 & 97.30 \\
\hline C10 & 164.2 & 165.8 & -1.6 & 164.61 \\
\hline C11 & 91.5 & 90.7 & 0.9 & 89.48 \\
\hline C12 & 158.0 & 159.1 & -1.1 & 157.76 \\
\hline C13 & 104.1 & 104.7 & -0.6 & 105.15 \\
\hline
\end{tabular}




\begin{tabular}{|r|c|c|c|c|}
\hline C14 & $\mathbf{1 9 0 . 4}$ & $\mathbf{1 9 3 . 8}$ & $\mathbf{- 3 . 4}$ & $\mathbf{1 9 2 . 4 9}$ \\
\hline C15 & 13.1 & 14.8 & -1.7 & 14.25 \\
\hline C18 & 55.3 & 56.7 & -1.4 & 56.68 \\
\hline C21 & $\mathbf{5 9 . 6}$ & $\mathbf{5 7 . 3}$ & $\mathbf{2 . 3}$ & $\mathbf{5 7 . 0 1}$ \\
\hline C23 & 58.6 & 57.3 & 1.3 & 56.39 \\
\hline${ }^{*} C^{*}$ & $194.4^{\text {sh }}$ & $195.0^{\text {sh }}$ & - & - \\
\hline${ }^{*} C^{*}$ & $90.7^{\text {sh }}$ or 94.6 & 90.7 or 93.2 & - & - \\
\hline
\end{tabular}

sh - shoulder to the peak

${ }^{\star} C^{*}$ - refers to the additional peaks 